\title{
Laser Doppler Sensing for Blood Vessel Detection with a Biologically Inspired Steerable Needle
}

\author{
V Virdyawan ${ }^{1}$, M Oldfield ${ }^{2}$ and F Rodriguez y Baena ${ }^{1}$ \\ ${ }^{1}$ Mechanical Engineering Department, Imperial College London, London SW7 2AZ, \\ UK \\ ${ }^{2}$ Department of Mechanical Engineering Sciences, University of Surrey, Guildford \\ GU2 7XH, UK
}

E-mail: f.rodriguez@imperial.ac.uk

\begin{abstract}
Puncturing blood vessels during percutaneous intervention in minimally invasive brain surgery can be a life threatening complication. Embedding a forward looking sensor in a rigid needle has been proposed to tackle this problem but, when using a rigid needle, the procedure needs to be interrupted and the needle extracted if a vessel is detected. As an alternative, we propose a novel optical method to detect a vessel in front of a steerable needle. The needle itself is based on a biomimetic, multi-segment design featuring four hollow working channels. Initially, a laser Doppler flowmetry probe is characterized in a tissue phantom with optical properties mimicking those of human gray matter. Experiments are performed to show that the probe has a $2.1 \mathrm{~mm}$ penetration depth and a $1 \mathrm{~mm}$ off-axis detection range for a blood vessel phantom with $5 \mathrm{~mm} / \mathrm{s}$ flow velocity. This outcome demonstrates that the probe fulfills the minimum requirements for it to be used in conjunction with our needle. A pair of Doppler probes is then embedded in two of the four working channels of the needle and vessel reconstruction is performed using successive measurements to determine the depth and the off-axis position of the vessel from each laser Doppler probe. The off-axis position from each Doppler probe is then used to generate a "detection circle" per probe, and vessel orientation is predicted using tangent lines between the two. The vessel reconstruction has a depth Root Mean Square Error (RMSE) of $0.3 \mathrm{~mm}$ and an RMSE of $15^{\circ}$ in the angular prediction, showing real promise for a future clinical application of this detection system.
\end{abstract}

PACS numbers: 87.85.st

Keywords: Laser Doppler, Steerable Needle, Blood Vessel Detection

Submitted to: Bioinspir. Biomim. 


\section{Introduction}

In minimally invasive brain surgery, percutaneous interventions are commonly performed, for example, in biopsies [1], deep brain stimulation (DBS) implantation [2], and a relatively new direct drug infusion method to the brain called Convection Enhanced Delivery (CED) [3]. During these procedures, inadvertently puncturing blood vessels can be a life-threatening complication $[4,5,6,7,8,9]$. In [5], up to $8 \%$ of patients who underwent the biopsy procedure had this complication, while during deep brain stimulation, [6] found an occurrence of 5\%. Even though preoperative imaging data are used to plan a safe, obstacle-free path, intraoperatively, brain shift and other tissue deformations (such as pulsatile motion and breathing) may occur that would invalidate the plan, with the subsequent need for updated images to be acquired [10]. In order to avoid these complications, real time sensing is needed in the operating theater. Unfortunately, commonly used imaging modalities such as Magnetic Resonance Imaging (MRI), X-Ray and Ultrasound do not lend themselves for use intraoperatively in this application. MRI is expensive, requires significant processing time/computational power to construct the image volume and requires MRI-compatible tools [11, 12]. The ionizing radiation of X-rays hinders its application in lengthy surgical procedures $[11,12]$. Finally, while ultrasound can and has been used intraoperatively, its resolution is currently not able to capture millimeter-size vessels, and ultrasound waves cannot easily penetrate the skull [13].

To avoid a hemorrhage caused by puncturing a blood vessel during a procedure, investigations have been conducted on the deployment of forward-looking, laser-based sensors mounted on the needle tip. The laser signals are delivered and collected through optical fibers. Liang et. al [14] used an Optical Coherence Tomography (OCT) probe as the sensor to detect a blood vessel in front of the advancing needle. In [13], a CoherenceGated Doppler (CGD) system was developed that detected a blood vessel deeper into brain tissue, compared to OCT. However, the CGD system lacked quantitative information on the position of the vessel. The only existing literature describing a real clinical procedure is that from Wardell et. al. [15], where a commercial Laser Doppler Flowmetry (LDF) probe is used as a potential vessel tracking method to record the perfusion value along the insertion path during DBS implantation.

Additionally, current percutaneous intervention procedures use a rigid needle. This means that, if there is an obstacle (e.g. a blood vessel) detected along the insertion path, the procedure must be interrupted. To address this limitation, current research has been directed towards the field of steerable needles, as these have the ability to steer around obstacles and thus correct for any misalignment [16]. Steering inside tissue is achieved by controlling the bending of the needle either as a direct result of passive needle-tissue interactions or active modification of the needle shape [17]. Steerable needle designs cover bevel-tipped needles [18], concentric tube needles [19], tendon actuated needles $[20,21]$, and programmable bevel needles [22]. Spinning the needle about its insertion axis is required to control deflection of bevel-tipped needles. However, this type of 
spinning can cause damage to the surrounding tissue during insertion [18]. The tip of the tendon-actuated needle can be articulated during the insertion to control tool-tissue interaction forces and thus the insertion trajectory, but, in doing so, it will displace the tissue surrounding the tip of the needle [21], which is undesirable. Steering concentric tube needles does not require tool-tissue interaction forces to be exploited, but, for use as a steerable needle system, follow-the-leader deployment is necessary, which continues to present significant technical challenges [23].

Although any steerable needle system has the ability to avoid an obstacle, to date there is no reported use of a steerable needle system with embedded forward looking sensors to avoid a vessel along its path. Indeed, the main focus areas for steerable needle sensing to date have been position tracking, force sensing, and shape sensing [18, 24, 25, 26]. Ayvali et. al [27] studied the use of OCT in an active needle system. They showed that the OCT probe developed by Liang et. al. [14] could be used in a bending needle. However, in [27] no study about vessel avoidance using their needle system was presented.

The programmable bevel-tip needle (PBN) design, first demonstrated by Frasson et. al in 2008 [28] and originally referred to as "Soft Tissue Intervention and Neurosurgical Guide (STING)", offers a multi-segment approach, which is inspired by the ovipositor of parasitic wasps in the Ichneumonoidea family. The ovipositor, which is approximately $0.2 \mathrm{~mm}$ in diameter, can be as long as $18 \mathrm{~cm}$, possessing the ability to penetrate wood and to steer within it to reach a suitable location to lay eggs [29]. It consists of multiple valves, called ventral and dorsal valves, which interlock with one another through a dovetail mechanism called the olistheter [29]. The olistheter holds the valves together, but also allows them to slide independently with respect to one another. This unique configuration enables the insect to enact a reciprocating insertion strategy, in which forward axial motion is performed sequentially by each valve, in such a way to avoid buckling even in the presence of a hard substrate, such as the bark of a tree $[30,31]$. Outward facing teeth are present at the tip of the valves, which can be used to anchor each segment onto the wood to generate a tensioning force that stabilizes the insertion [31]. After failing to reproduce this mechanism based on anisotropic surface characteristics [28], the PBN design evolved into the form described here. Teeth are replaced by a complex sequential insertion method that achieves the same tensioning effect through the ratio between stationary and moving segments of a 4-part PBN (i.e. always maintaining a larger amount of contact surface that is stationary at any one point during the insertion). We have demonstrated in previous work that such an approach enables us to reduce surrounding tissue motion during an insertion, thus increasing the accuracy of the needle tip delivery [32].

Detailed observation of a parasitic wasp probing behavior shows that the multi-segment ovipositor is also useful for steering [33]. The offset between segments generates an asymmetric reaction force in the tip of the ovipositor during the insertion process [33] that results in bending along a prescribed direction (Figure 1). By varying this asymmetry, the radius of curvature of the bend can be controlled, enabling the ovipositor to be steered [33]. The ovipositor also possesses "sensilla", which provide sensory 
feedback about the movement of the ovipositor [29] or to discriminate the type of the substrate being traversed [34].

Similar to its biological counterpart, our current PBN design consists of at least two segments that are linked by a dovetail mechanism, enabling these to slide with respect to one another. Embodiments of a four-segment needle demonstrate the ability to steer along an arbitrary three-dimensional (3D) path along 8 principal directions by changing the leading segment [35] (Figure 2c) without relying on transmission of torque from base to tip. Therefore, PBNs can be manufactured from highly compliant materials that can be matched to a softer tissue, such as brain, in order to enhance dexterity [36]. In addition, compliant materials can also be useful to manufacture an implantable needle for long term brain treatment [37], a clear focus of the current, EU funded EDEN2020 project (www.eden2020.eu).

The current embodiment of the needle is manufactured using a biocompatible polymer with an outer diameter of $2.5 \mathrm{~mm}$, which is the same size as that of commercially available biopsy needles (e.g. the sedan side cutting biopsy needle, A2430-01, Electa, Stockholm, Sweden). Figure 2 shows microscope images of the PBN, a representation of the relative motion between segments, and a schematic of its cross section. Figure 2c shows that the needle has a lumen in each of its segments. The lumen can be used as a conduit to deliver drugs and minimally invasive instruments, or as the working channel for imaging modalities based on optical fibers.

Even though several aspects of the PBN have been studied [28, 38, 39, 40], this paper captures our first attempt at making use of the unique capabilities of the system to solve the clinical need to avoid hemorrhages, with the proof of concept of a detection method based on measurements from forward looking optical sensors embedded within the steerable needle segments. By using two sensors in two of the four working channels of a PBN prototype, we demonstrate that vessel pose can be inferred, providing the necessary knowledge to plan and execute a subsequent avoidance strategy, a method that could also be applied to other needle designs, for instance the wasp-inspired steerable needle in [41].

the paper is organized as follows. Firstly, a commercial laser Doppler blood-flow monitoring system is experimentally characterized in a brain tissue phantom. Based on the characterization results, a feasibility study where this probe is deployed within the $\mathrm{PBN}$ is then conducted. Secondly, an algorithm is presented, which enables prediction of the vessel orientation and position using two sensors embedded within the needle,

with experimental results using the proposed algorithm discussed. The paper concludes with a summary of the main findings, where a number of future research avenues are highlighted.

\section{Laser Doppler Flowmetry Characterization}

Laser Doppler Flowmetry (LDF) monitoring systems are commonly used to monitor microcirculation (i.e. circulation in very small vascular capillaries). The LDF sensors 


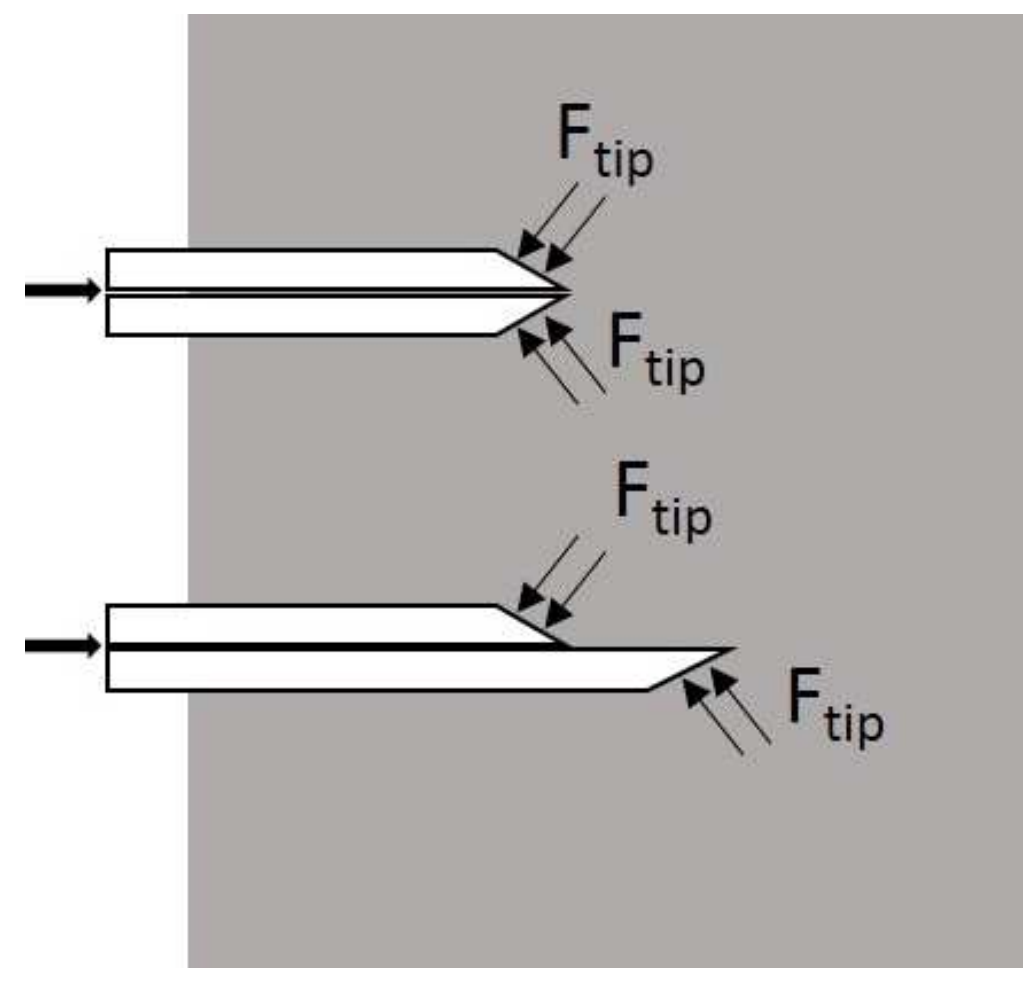

Figure 1: The tip force is symmetric where the segments are aligned so that there is no bending in the ovipositor, the asymmetric force where the ovipositor has an offset produces bending

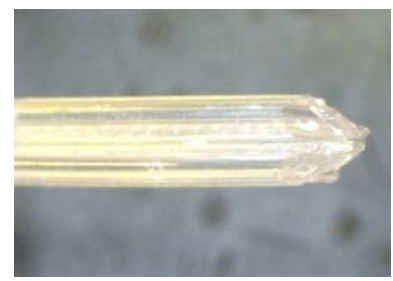

(a)

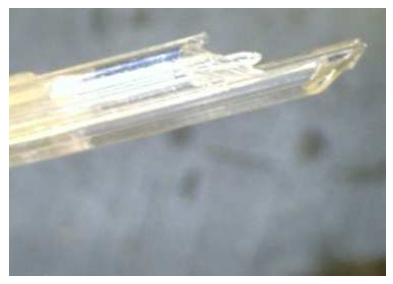

(b)

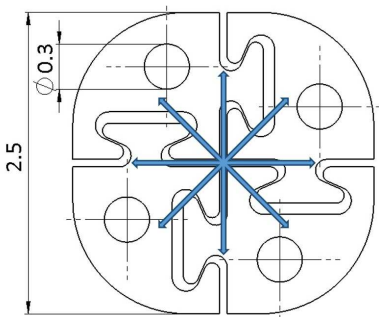

(c)

Figure 2: (a) The PBN with all of the segments aligned, and (b) with offsets introduced into the needle. (c) Cross section of the needle, which has a diameter of $2.5 \mathrm{~mm}$ and four, $0.3 \mathrm{~mm}$ OD working channels. Steering direction can be controlled by changing leading segment, the arrows indicate eight principal steering directions [35] 
detect blood vessels by measuring the Doppler shift generated by the movement of the blood cells that they contain. Since light is scattered inside the substrate, it is challenging to determine the source of the signals. This scattered light means that determining the absolute perfusion value of blood flow is not possible [42]. Brownian motion of latex particles in water is used as a standard to calibrate an LDF system [43]. Therefore, the perfusion measurement is a relative value with an arbitrary unit (AU). Based on the simulation results of Fredrikson et. al. [44], the measurement range of LDF possesses a larger off-axis detection range compared to OCT and CGD (up to a few $\mathrm{mm}$ for LDF, $10 \mu \mathrm{m}$ for OCT, and $40 \mu \mathrm{m}$ for CGD). Consequently, a laser Doppler system (OxyFlo ${ }^{\mathrm{TM}}$, Oxford Optronix, Abingdon, UK), with a bare-fiber type probe (NX-BF/F, Oxford Optronix, Abingdon, UK) was chosen as the sensor (Figure $3 \mathrm{~b}$ ), since an offset exists between the center of the PBN lumens and the center of the needle itself (see Figure 2c). To simplify the setup process, in this study, LDF is used to detect the presence of larger vessels, with a diameter $>0.2 \mathrm{~mm}$ [45]. The Doppler probe that was used consists of two fibers, one for illumination and the other for collection of the light (Figure 3a). In Fredriksson et. al. [44] it was shown that, for 0 to $1.2 \mathrm{~mm}$ fiber separation (i.e. the center to center distance between fibers), the larger the separation between source and collector fibers, the deeper the source of the reflected signals that could be detected. Due to the lumen size of each needle segment ( $0.30 \mathrm{~mm}$, Figure $2 \mathrm{c})$, the probe that was used during the experiments also has a $0.30 \mathrm{~mm}$ outer diameter. Both the illumination and collection fibers have a diameter of $0.15 \mathrm{~mm}$, with $0.15 \mathrm{~mm}$ fiber separation. To verify the use of the probe as a vessel detector able to inform an eventual puncture avoidance system, the probe should have the ability to detect a vessel located in front of the tip of the needle. Based on the geometry of the tip, Figure 4 shows the minimum detection range for the sensor to satisfy this requirement $(>0.67$ $\mathrm{mm}$ depth detection, and $>0.88 \mathrm{~mm}$ off-axis detection).

\subsection{Characterization Materials and Methods}

The sensing capabilities of the probe were tested using a phantom that mimicked the optical properties of a blood vessel inside human gray matter. In order to achieve the $0.75 \mathrm{~mm}^{-1}$ reduced scattering coefficient of human gray matter [46], $3 \mathrm{~g} / \mathrm{L}$ of titanium dioxide powder ( $\mathrm{TiO} 2$ ) [47] was added to $4.5 \%$ weight gelatin, achieving a measured Young's Modulus of $7 \mathrm{kPa}$ [48], which is in the ballpark of measurements taken for gray matter [49]. A capillary tube with an outer diameter of $0.9 \mathrm{~mm}$ and an inner diameter of $0.6 \mathrm{~mm}$ was used as a blood vessel phantom, while the blood surrogate was milk, with $1.5 \%$ fat content [50]. In this paper, the term depth detection is defined as the distance between the probe and the outer surface of the tube, while the off-axis (lateral) distance is defined as the distance between the center axis of the tube and the center axis of the probe. To test the measurable depth of a vessel below the gelatin surface, the capillary tube was placed at a gradient $\left(\theta_{v}\right)$ inside the gelatin. The maximum depth detection range was investigated by scanning axially above the tube on the gelatin surface (Figure 


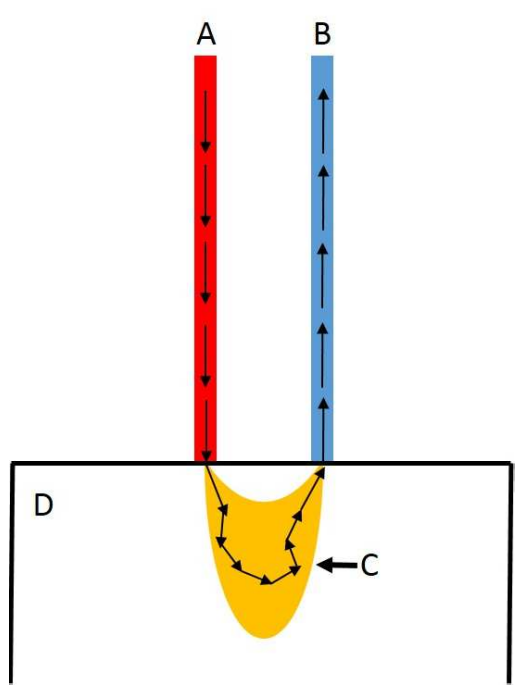

(a)

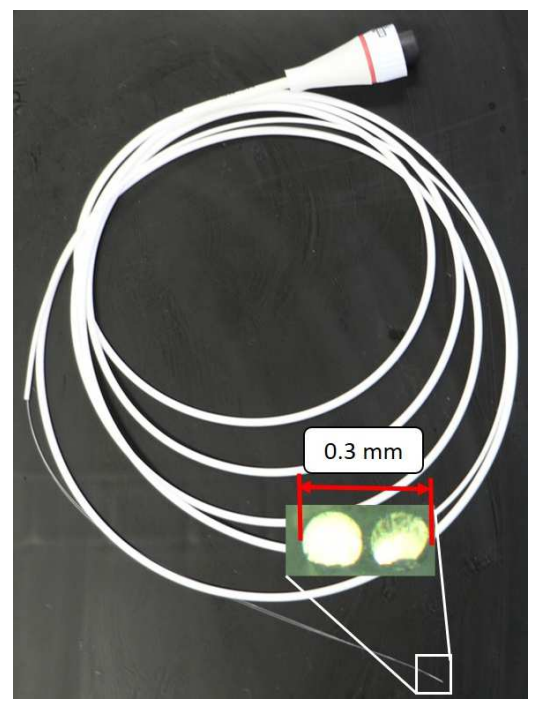

(b)

Figure 3: a) LDF probe schematic diagram. Light is delivered through an illumination fiber, scattered in the substrate and collected using a collection fiber, b) a bare-fiber type probe (NX-BF/F, Oxford Optronix, Abingdon, UK) with its corresponding cross-section (inset). A:Illumination fiber, B: Collection fiber, C: Scattered light, D: substrate

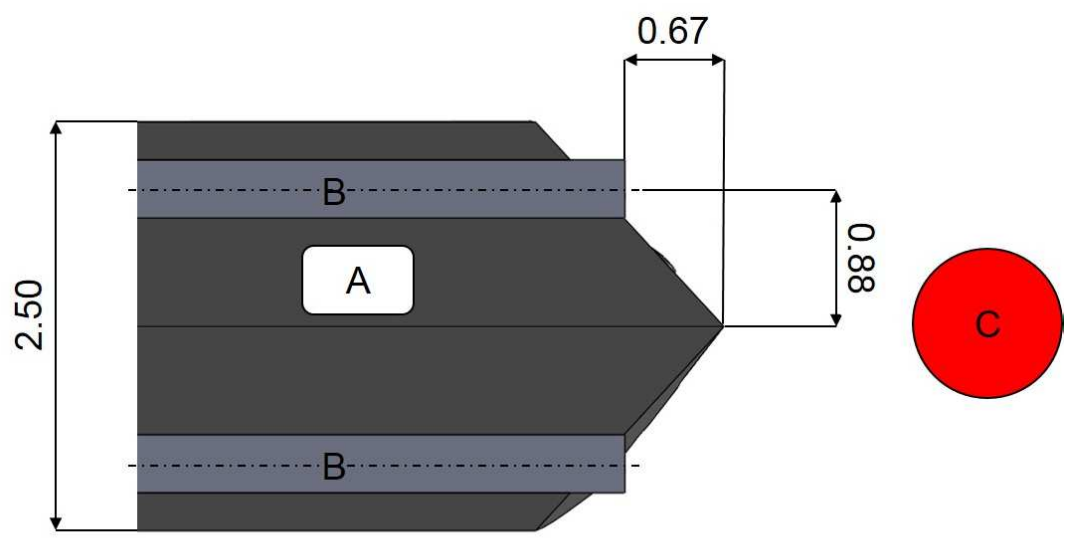

Figure 4: Minimum requirements for the detection range of the probe that is used for vessel detection in the PBN. A: the PBN, B: Optical fibers, C: Blood vessel 


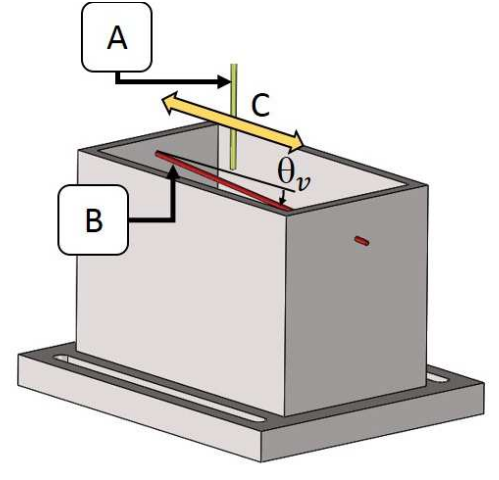

(a)

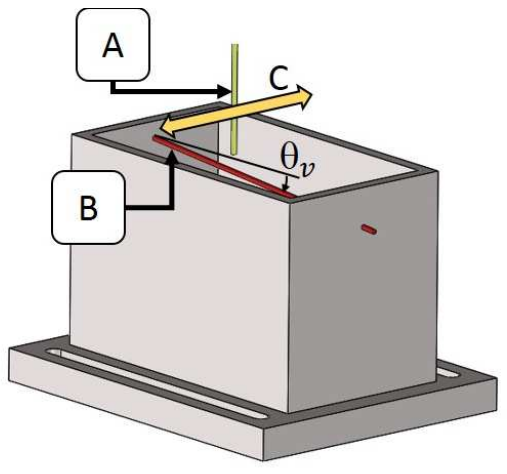

(b)

Figure 5: Schematic diagram of the characterization procedure for: a) depth detection range, b) off-axis detection range. A: LDF probe, B: blood vessel phantom, C:direction of movement, $\theta_{v}$ : the angle of the vessel phantom

5a). The off-axis detection range was measured by moving the probe laterally across the tube's axis (Figure 5b). The Doppler probe was held inside a capillary tube in a two-degree-of-freedom precision linear stage (Figure 6).

During the experiments, milk flow velocities were achieved using a syringe pump (Graseby 3200, Graseby Medical Ltd., UK). The depth detection range was measured for three different flow velocities, $1 \mathrm{~mm} / \mathrm{s}, 5 \mathrm{~mm} / \mathrm{s}$ and $10 \mathrm{~mm} / \mathrm{s}$, with $0.30 \mathrm{~mm}$ depth increments, starting from $0.30 \mathrm{~mm}$ tube depth. Lateral measurements were only performed for $5 \mathrm{~mm} / \mathrm{s}$ flow velocity, which represents the maximum flow velocity described in [13], with $0.10 \mathrm{~mm}$ resolution and at four different tube depths $(0.30 \mathrm{~mm}$, $0.90 \mathrm{~mm}, 1.50 \mathrm{~mm}$, and $2.10 \mathrm{~mm}$ ). In order to calibrate the zero depth position, the sensor was moved along the gelatin surface (right to left, Figure 5a) until it touched the tube. Since the tube was placed manually in the gelatin box, the computed uncertainty in finding the zero depth position was estimated as $\pm 0.10 \mathrm{~mm}$. In each position, the Doppler signal was measured a minimum of 5 times and the average was taken.

\subsection{Characterization Results}

Figure 7 shows the perfusion value for different flow velocities at various depths. Figure 8 shows the off-axis perfusion value for a flow velocity of $5 \mathrm{~mm} / \mathrm{s}$ at four depth positions when measured across the capillary tube. The background signal of the LDF system was measured when there was no flow in the capillary tube and was found to fluctuate (mean: 13.7 AU, standard deviation: 29.4 AU). The threshold for vessel detection was empirically set at 120 AU since, starting from this value, the perfusion level showed a statistically significant difference for all depths, when compared to the lowest 


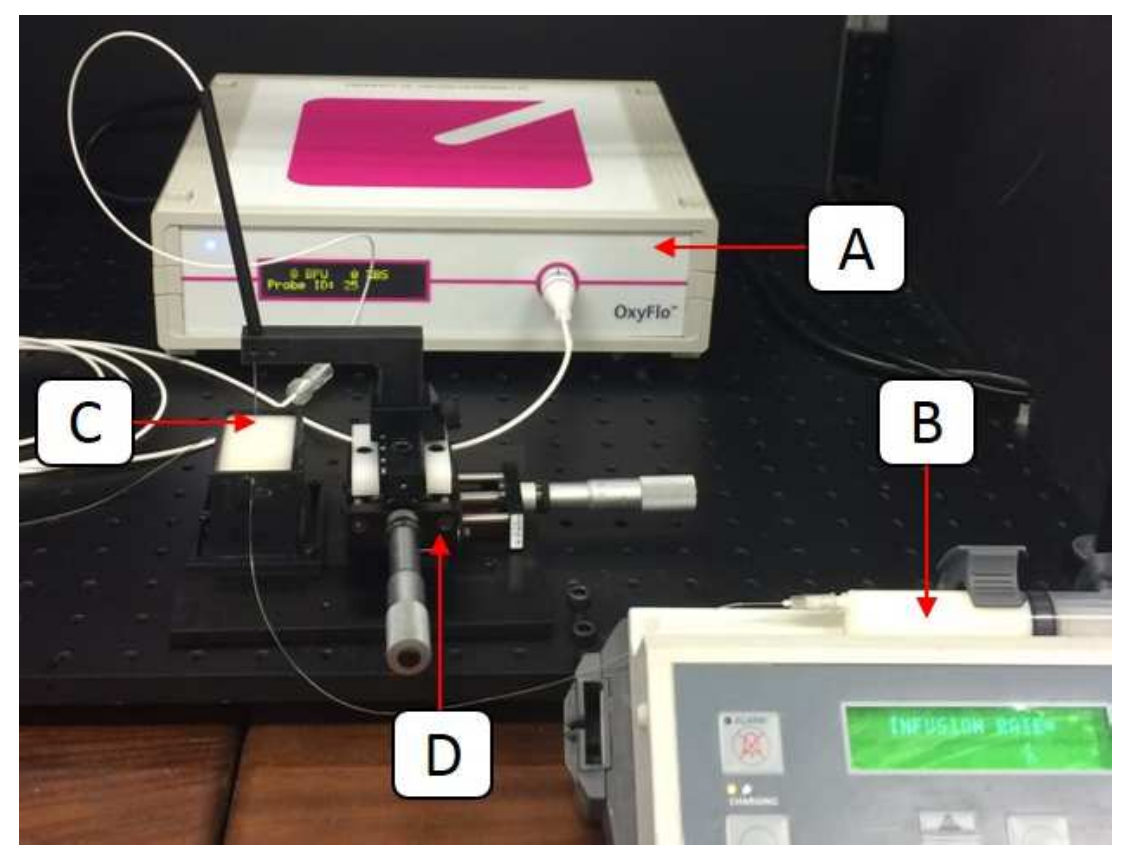

Figure 6: Experimental configuration for laser Doppler characterization of vessel flow in a brain phantom, using a commercially available laser Doppler system (OxyFlo ${ }^{\mathrm{TM}}$ (Oxford Optronix, Abingdon, UK). A: LDF system, B: perfusion pump, C: LDF probe, D: linear stage

measurement value at $2.00 \mathrm{~mm}$ off-axis position. Using this threshold, the maximum detection depth for a $5 \mathrm{~mm} / \mathrm{s}$ flow velocity was found to be $2.10 \mathrm{~mm}$. Using the same setup, measurement thresholds for $1.20 \mathrm{~mm}, 1.00 \mathrm{~mm}, 0.50 \mathrm{~mm}$, and $0.30 \mathrm{~mm}$ off-axis positions of the Doppler probe were found to be $0.30 \mathrm{~mm}, 0.90 \mathrm{~mm}, 1.50 \mathrm{~mm}$, and $2.10 \mathrm{~mm}$ depth, respectively. For the same position, $10 \mathrm{~mm} / \mathrm{s}$ flow velocity gives a higher perfusion value compared to $5 \mathrm{~mm} / \mathrm{s}$. Consequently, the maximum detection depth also increases up to $2.70 \mathrm{~mm}$. A lower, $1 \mathrm{~mm} / \mathrm{s}$ flow velocity also gives a lower perfusion value, with maximum detection depth of only $0.90 \mathrm{~mm}$. Comparing these characterization results to the characteristics of our needle (Figure 4), the minimum detection range of $0.67 \mathrm{~mm}$ at $0.88 \mathrm{~mm}$ off-distance for blood detection is fulfilled for flow velocities above $5 \mathrm{~mm} / \mathrm{s}$.

\section{Vessel position and Orientation Prediction - Proof of Concept}

In this section, a new method to predict the position and orientation of a possible vessel in front of the needle is proposed. The detection algorithm is based on the characterization results for $5 \mathrm{~mm} / \mathrm{s}$ flow velocity (Section 2). These characterization results show that, for a given perfusion value, the exact position and orientation of 


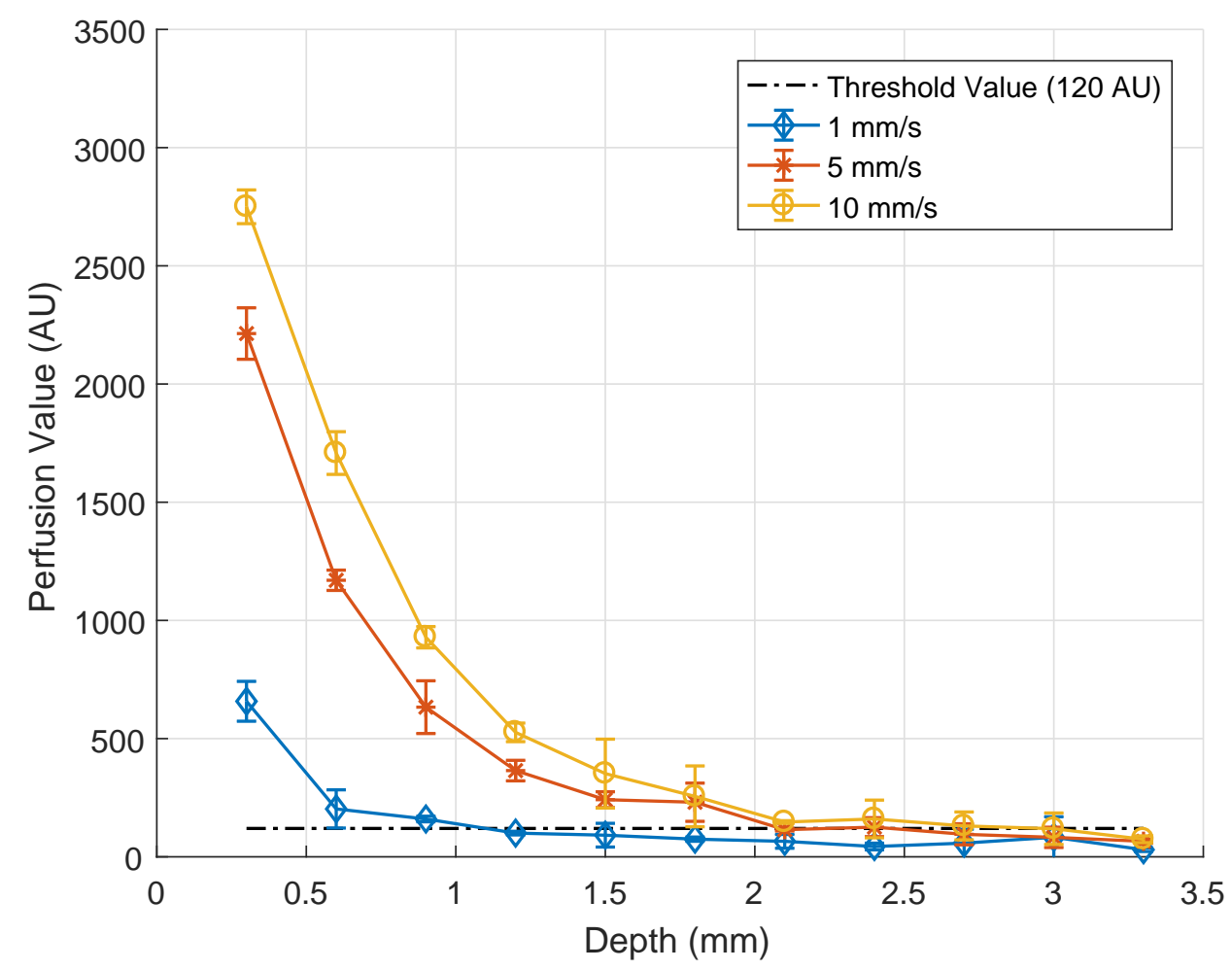

Figure 7: Mean and standard deviations of perfusion value against capillary depth for three different flow velocities, the dash-dot line is the threshold value

a vessel cannot be determined using a single measurement, since positions that give the same perfusion value are not unique. Successive measurements, combined with measurements from multiple optical probes made possible due to the PBN's multisegment design, were used to infer the tube position and orientation. For the algorithm developed here, we make the assumption that the vessel detected by the probe is just one and that it is lying approximately in a plane normal to the needle axis.

\subsection{Materials and Methods}

3.1.1. Insertion Experiments using Two LDF Probes The same tissue and blood vessel phantom was used as for the characterization experiments (Section 2.1). A tube was placed inside a gelatin box on a gradient (Figure 9a). Two laser Doppler probes were placed in the PBN prototype, as shown in Figure 9b. The needle, embedded with the probes, was then placed in a two degree-of-freedom linear stage. To investigate the ability of the probes to discriminate between vessel poses, measurements were taken for several tip off-axis positions (defined with respect to the needle coordinate system $\left(x_{n}, y_{n}, z_{n}\right)$ in which the axis of the vessel intersects with $\left.x_{n}\right)$. The center of the global coordinates of the box was set at $0.00 \mathrm{~mm}$ tip off-axis position. By moving the PBN in the $y$ direction, a variable tip off-axis distance could be created. Perfusion values 


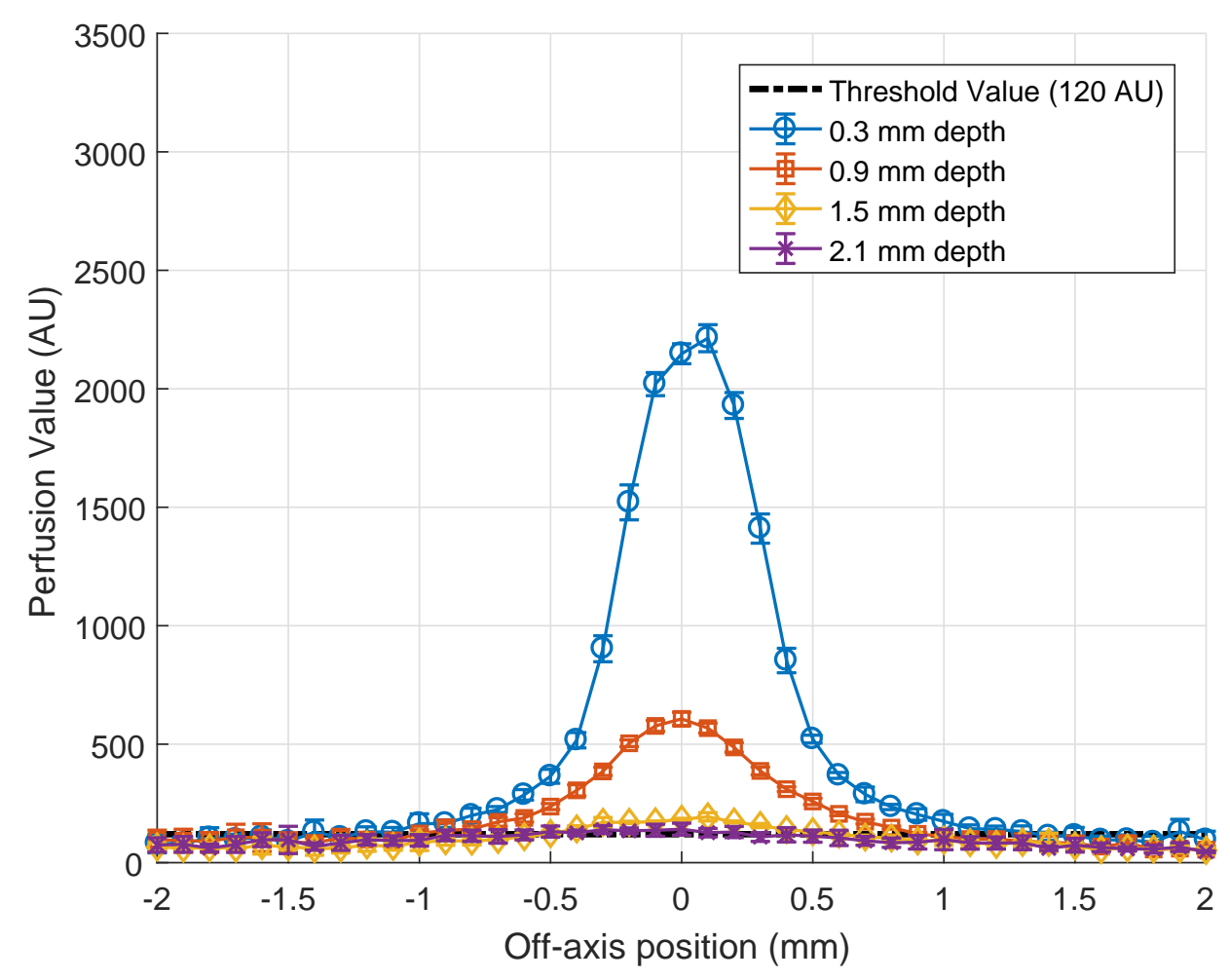

Figure 8: Mean and standard deviations for off-axis perfusion value when measured laterally across the capillary at different depths, the dash-dot line is the threshold value

from both of the probes were measured in five different tip off-axis positions: -0.35 $\mathrm{mm},-0.18 \mathrm{~mm}, 0.00 \mathrm{~mm}, 0.18 \mathrm{~mm}$, and $0.35 \mathrm{~mm}$. At each tip off-axis position, the needle was advanced in the positive $z$ direction at $0.50 \mathrm{~mm}$ increments, as in Wardell et. al. [15], since it is the minimum resolution needed to be able to detect local changes in microvascular measurements, starting $3.00 \mathrm{~mm}$ away from the tangent plane of the vessel in the $x y$-plane. This starting location was set as $z=0$. During all insertions, the flow velocity was maintained at $5 \mathrm{~mm} / \mathrm{s}$. For every depth increment, the needle was kept in place and the Doppler signal was measured during that time, alternating between the two probes ( $5 \mathrm{~s}$ for each probe) due to the inability of the detection system chosen here to look at both simultaneously. Ten repetitions were performed for each tip off-axis position. The experimental set-up for predicting the tube position and orientation is shown in Figure 10.

\subsubsection{Algorithm Development for Vessel Position and Orientation Prediction To} develop the algorithm for predicting the vessel position and orientation (i.e. the pose), the characterization results from Section 2.2 were approximated using a twodimensional function. To find the best approximation, off-axis and depth measurements were analyzed individually before being combined into a single expression. The steps to find the approximating function were as follows: 


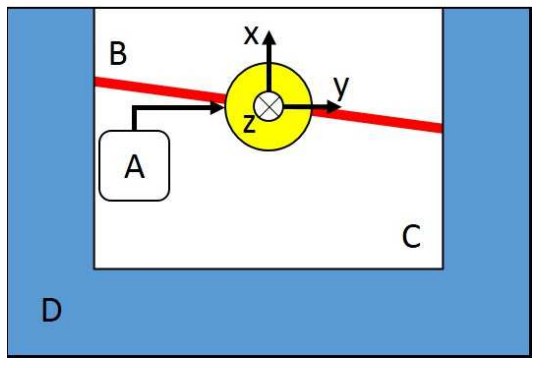

(a)

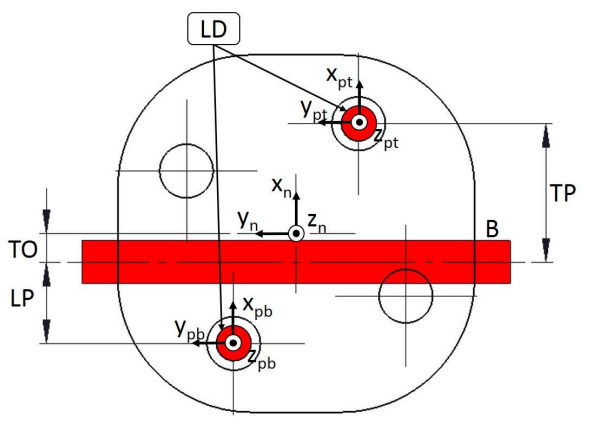

(b)

Figure 9: (a) A schematic diagram of vessel detection experiments with a global coordinate system. (b) The position of two laser Doppler probes in the PBN with the definitions and terminology used for predicting the vessel; three local coordinates at the tip of the needle $\left(x_{n}, y_{n}, z_{n}\right)$, the bottom probe $\left(x_{p b}, y_{p b}, z_{p b}\right)$, and the top probe $\left(x_{p t}, y_{p t}, z_{p t}\right)$ were used. A: The PBN, B: vessel phantom, C:tissue phantom, D: Gelatin box, LD: laser Doppler probe, TO: tip off-axis distance, TP: top probe off-axis distance, LP: bottom probe off-axis distance

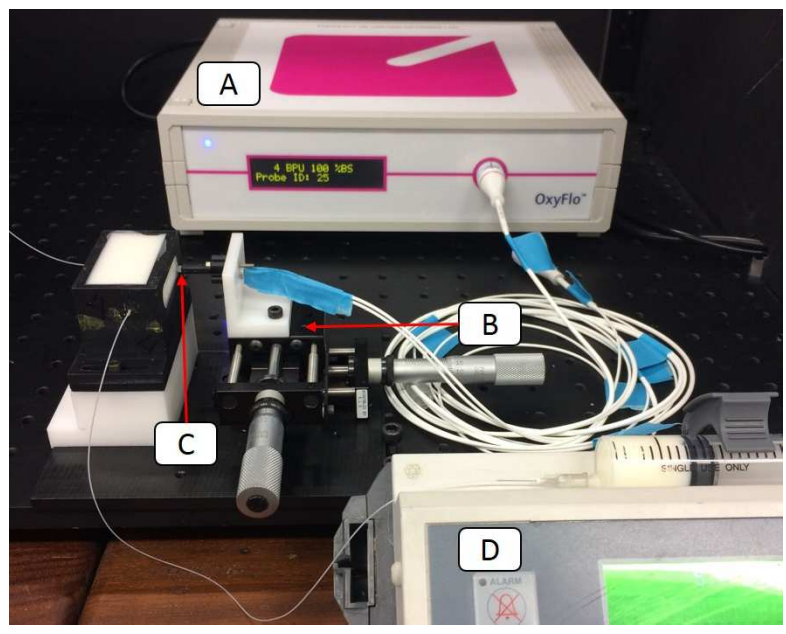

Figure 10: Insertion configuration for vessel prediction experiments, the LDF system used here was the same single channel LDF system as in Section 2. A: LDF system, B: linear stage, C: the PBN, D: syringe pump 


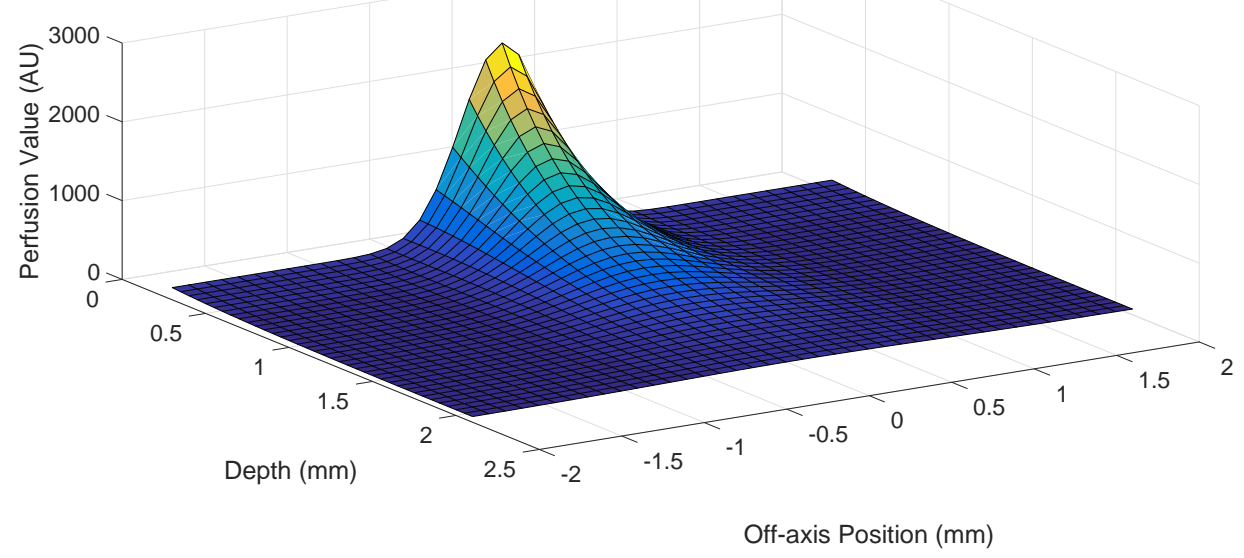

Figure 11: 2D map of the perfusion value generated using (1)

- The depth perfusion value is approximated using an exponential function since the probability of a transmitted photon is also an exponential function [51].

- The off-axis perfusion value for each depth is approximated by a bell shaped function with the Gaussian $f_{z i}(y)=a_{0} \mathrm{e}^{\left(-y^{2} / a_{1}^{2}\right)}$, where $a_{1}$ is a variable used to account for off-axis perfusion values at different depths.

The complete behavior was thus approximated as:

$$
f\left(y_{p}, z_{p}\right)=\left(c_{1} \mathrm{e}^{\left(-y_{p}^{2} /\left(c_{2} \mathrm{e}^{\left(c_{3} z_{p}\right)}\right)^{2}\right)}+c_{4}\right) \mathrm{e}^{c_{5} z_{p}}+b
$$

where $b$ is background noise, $y_{p}$ is the off-axis distance from the laser Doppler probe to the vessel, and $z_{p}$ is the depth of the laser Doppler probe from the vessel. Constants $c_{1}-c_{5}$ were found empirically using the MATLAB (The Mathworks inc., USA) curve fitting toolbox. The two-dimensional (2D) map of perfusion values obtained from (1) is shown in Figure 11. LDF perfusion values for a given depth and off-axis position can be obtained from (1). However, during the insertion process, the depth $\left(z_{p}\right)$ and the off-axis $\left(y_{p}\right)$ position from the probe must be inferred from the measured signals using the inverse of (1). A lookup table of (1) facilitates its conversion into a grid used for mapping the insertion environment and provides the additional benefit of overcoming difficulties associated with an analytical inverse function, as the off-axis position, $y_{p}$, approaches zero.

Unlike a standard laser range finder that gives a measured distance directly, the LDF sensor does not provide a unique vessel position. There are multiple depths and off-axis positions that result in the same perfusion value. This is illustrated using the contour plot shown in Figure 12. To tackle this ambiguity, the recorded contour lines from each probe were transformed into global coordinates while the needle was advancing. A rigid glass tube was used as the blood vessel phantom, so an assumption could be made that the vessel in front of the needle would not move while the needle advanced towards it. Using this assumption, unique solutions were found at the intersection of two isolines in 


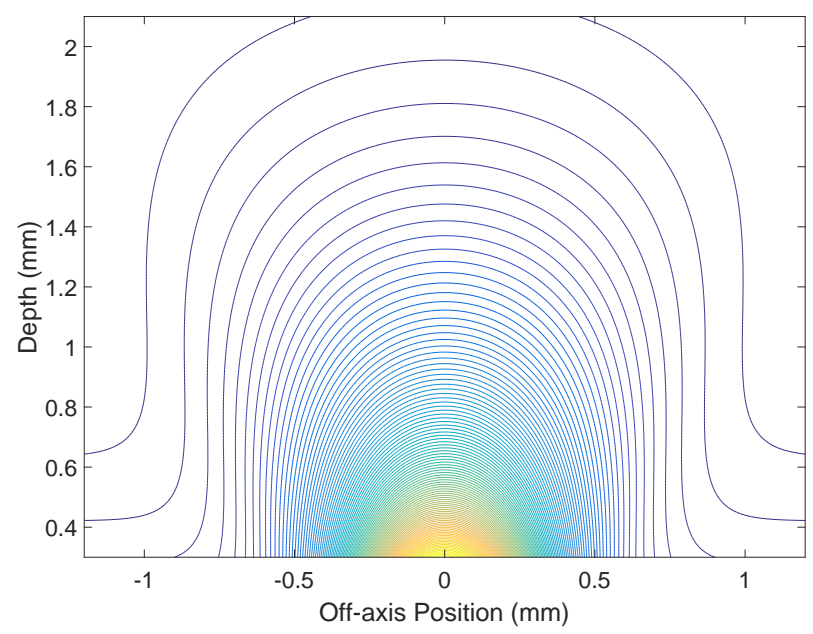

Figure 12: Contour plot of the LDF perfusion value measurements, each line showing depth and off-axis positions for the same perfusion value

the global coordinates of two incremental measurements. The contour lines show that there is a possibility of four intersection points (a pair at each of the two different $z$ positions). In order to reduce the solution to a pair at the same $z$ position, a minimum of three consecutive measurements is therefore required.

It should be noted that, up to this point, we assumed the vessel to be a line that lies on a plane parallel to the $x y$-plane perpendicular to the insertion axis of the needle (see Figure 9a). The location of this plane was at the $z$ position where the three contour lines intersect. To convert the $2 \mathrm{D}$ map into three dimensions, the probe measurement was assumed to possess rotational symmetry about the probe $z_{p}$ axis $\left(z_{p t}\right.$ for the top probe and $z_{p b}$ for the bottom probe) and normal to the vessel's assumed plane. This means that, in the vessel plane, the possible probe off-axis distance $\left(y_{p}\right)$ is rotated, generating a detection circle. The vessel position and orientation is then the tangent line of this detection circle, for which there are infinite solutions. To reduce the number of possibilities, measurements using the second embedded probe are used, as this measurement provides another detection circle. The vessel is then determined as coinciding with the tangent line to both of the circles, reducing the number of possibilities to four.

\subsection{Vessel Pose Prediction Results}

Figure 13 shows the mean and standard deviation of perfusion values for ten insertion experiments, using two laser Doppler probes, at five different tip off-axis positions. It shows that the position of the tube affects the perfusion value from each laser Doppler probe. At $0 \mathrm{~mm}$ tip off-axis position, there is no significant difference in perfusion values between the two probes, for all distances to the vessel. However, for the remaining tip off-axis positions, statistically significant differences in perfusion values ( $t$-test, $p=0.01$ ) 
are observed, starting from vessel positions which are $1.50 \mathrm{~mm}$ (Figure 13a and 13b) and $1.00 \mathrm{~mm}$ (Figure 13d and 13e) away from the tip of the needle.

The algorithm to predict vessel pose was used to analyze the data for each insertion. An example of how this algorithm works is shown in Figure 14, using the mean value measurements of the bottom probe at $0 \mathrm{~mm}$ tip off-axis position. A significant perfusion value ( $>120 \mathrm{AU})$ was detected at point $\mathrm{A}$ in Figure 14a that gives a corresponding contour line of possible vessel positions in the $x z$-plane in global coordinates, as shown in Figure 14b. At the next increment (point B) another contour line was recorded (Figure 14c). Even though, in this case, there already were a pair of intersection points at the same $z$ position, another measurement at point $\mathrm{C}$ was still required to account for the worst case scenario, where three successive measurements are required. After recording the perfusion value at Point $\mathrm{C}$, the third set of contour lines were recorded and the $z$ position of the vessel plane $(z=3.10 \mathrm{~mm})$ and the radius of the detection circle $(\mathrm{LP}=0.70 \mathrm{~mm}$ ) of the bottom probe were determined (Figure 14d). Repeating this method for the top probe then gave two detection circles. The possible number of vessel poses was then reduced to four, shown by dash-dot lines in Figure 15.

To investigate the performance of the algorithm, every insertion was analyzed. The characterization results (Section 2.2) show that, for a given depth and off-axis position, there is a variability in the LDF perfusion value. Therefore, in order to look for the corresponding contour line, the recorded value was included with \pm one standard deviation, as measured during the characterization process. The $z$ position of the vessel plane and radius of the detection circle in global coordinates were then determined by computing the centroid of the intersection area.

To quantify how good the tube reconstruction is, a comparison to the ground truth was conducted. Three parameters were compared:

- The $z$ position of the detected vessel

- The angle between the vessel and the tangent line between the two detection circles that lies closest to it, where the angle of the vessel was set experimentally to $2^{\circ}$ for all trials

- The $x_{n}$ intersection of the tangent line of the two detection circles that lies closest to the vessel (inset in Figure 15 (in this case, $x_{n}$ and $y_{n}$ align with $x$ and $y$ of the global coordinate)), which should equal to the real tip-off axis position of the vessel with respect to the needle tip.

Using the parameters above, the mean of the recorded depth position is $3.00 \mathrm{~mm}$, with $0.35 \mathrm{~mm}$ standard deviation and $0.37 \mathrm{~mm}$ RMS error. The RMS error for the tip off-axis prediction is $0.36 \mathrm{~mm}$. The angle prediction has an average of $5.8^{\circ}$, with $15.1^{\circ}$ standard deviation and $15.6^{\circ}$ RMS error. Tabulated data for these results is shown in Table 1. 


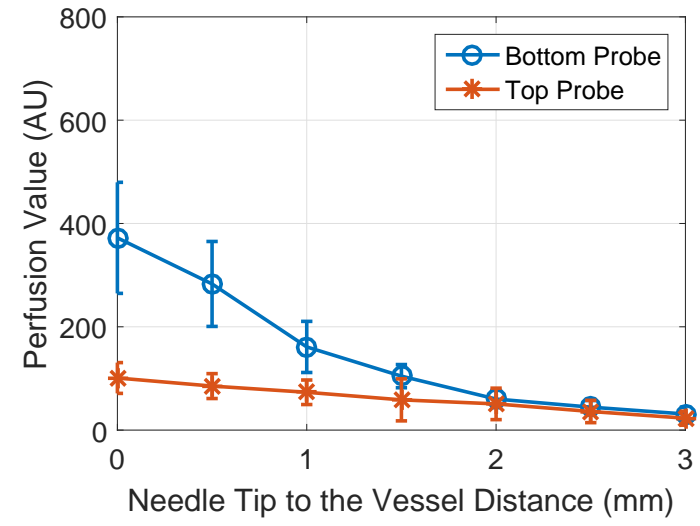

(a)

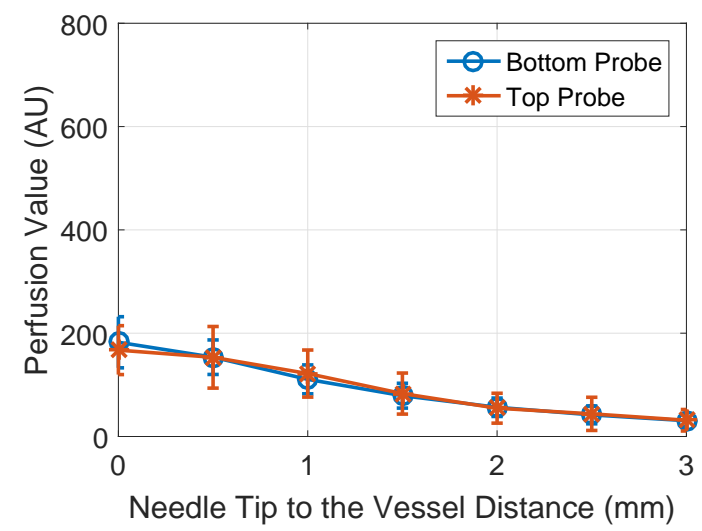

(c)

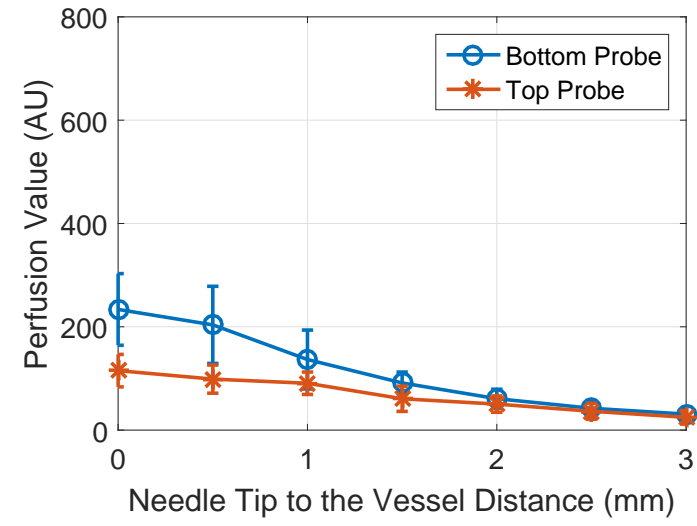

(b)

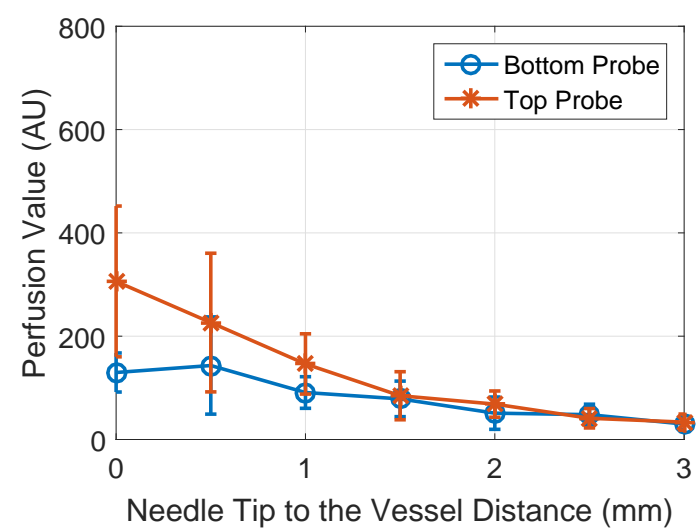

(d)

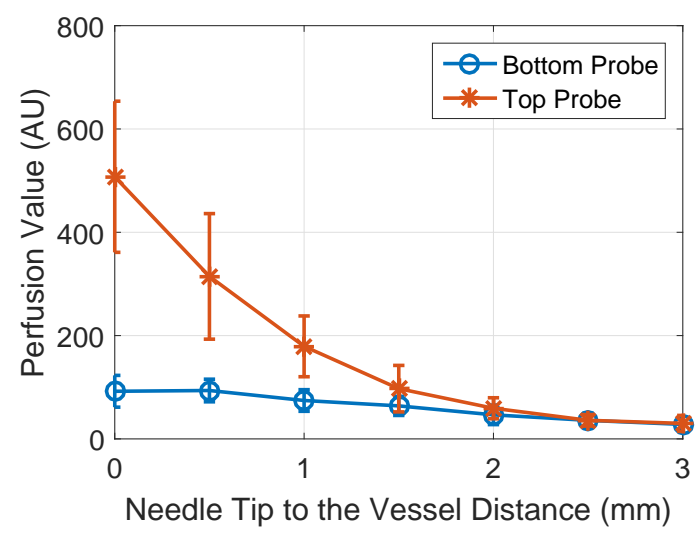

(e)

Figure 13: Mean and standard deviation of perfusion values for the top (asterisk) and bottom probes (circle), from $3.00 \mathrm{~mm}$ away from the surface of the needle, with 0.50 $\mathrm{mm}$ increments at a) $-0.36 \mathrm{~mm}, \mathrm{~b})-0.18 \mathrm{~mm}$, c) $0.00 \mathrm{~mm}$, d) $0.18 \mathrm{~mm}$, and e) $0.36 \mathrm{~mm}$ tip off-axis position 


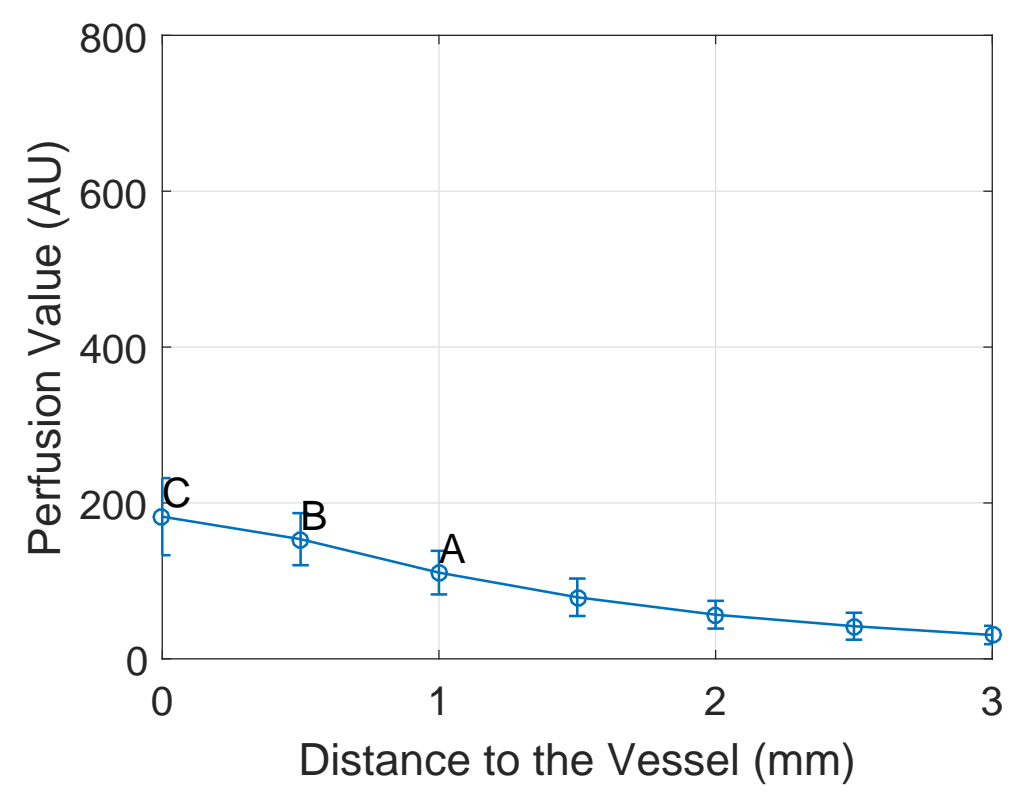

(a)

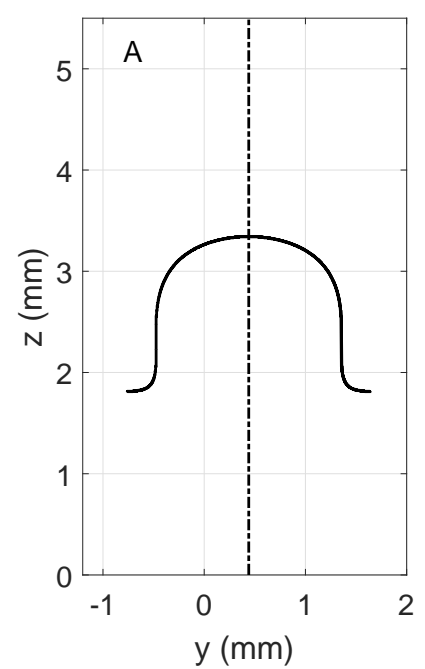

(b)

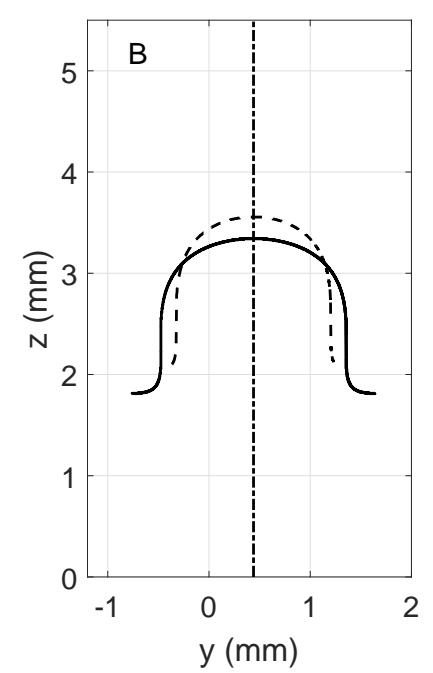

(c)

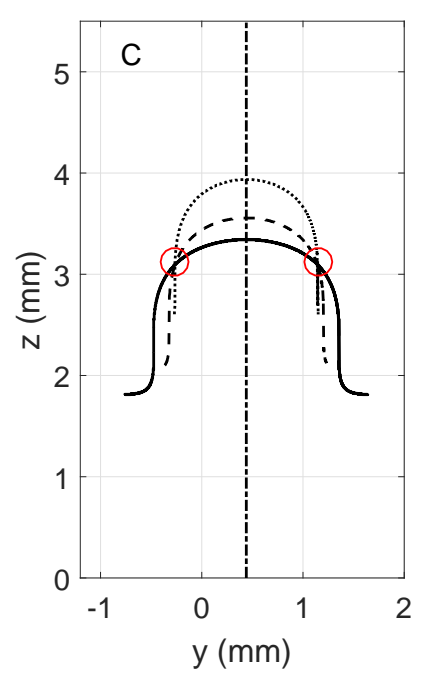

(d)

Figure 14: a) The mean perfusion value from the bottom probe (Figure 13c). The possible vessel position at point $\mathrm{A}, \mathrm{B}$, and $\mathrm{C}$ is shown in $\mathrm{b}$ ), c), and $\mathrm{d}$ ) respectively, the dash-dot line is the insertion axis of the laser Doppler probe. The circles in $\mathrm{d}$ ) show the possible vessel positions after three successive measurements

Table 1: Mean, standard deviation, and RMS error of the constructed vessel

\begin{tabular}{|c|l|r|r|r|}
\hline No & \multicolumn{1}{|c|}{ Parameter } & Angle $\left(^{\circ}\right)$ & Tip Off-axis $(\mathrm{mm})$ & $z$ position $(\mathrm{mm})$ \\
\hline 1 & Average & 5.8 & - & 3.00 \\
\hline 2 & Standard Deviation & 15.1 & - & 0.35 \\
\hline 3 & RMS Error & 15.6 & 0.36 & 0.37 \\
\hline
\end{tabular}




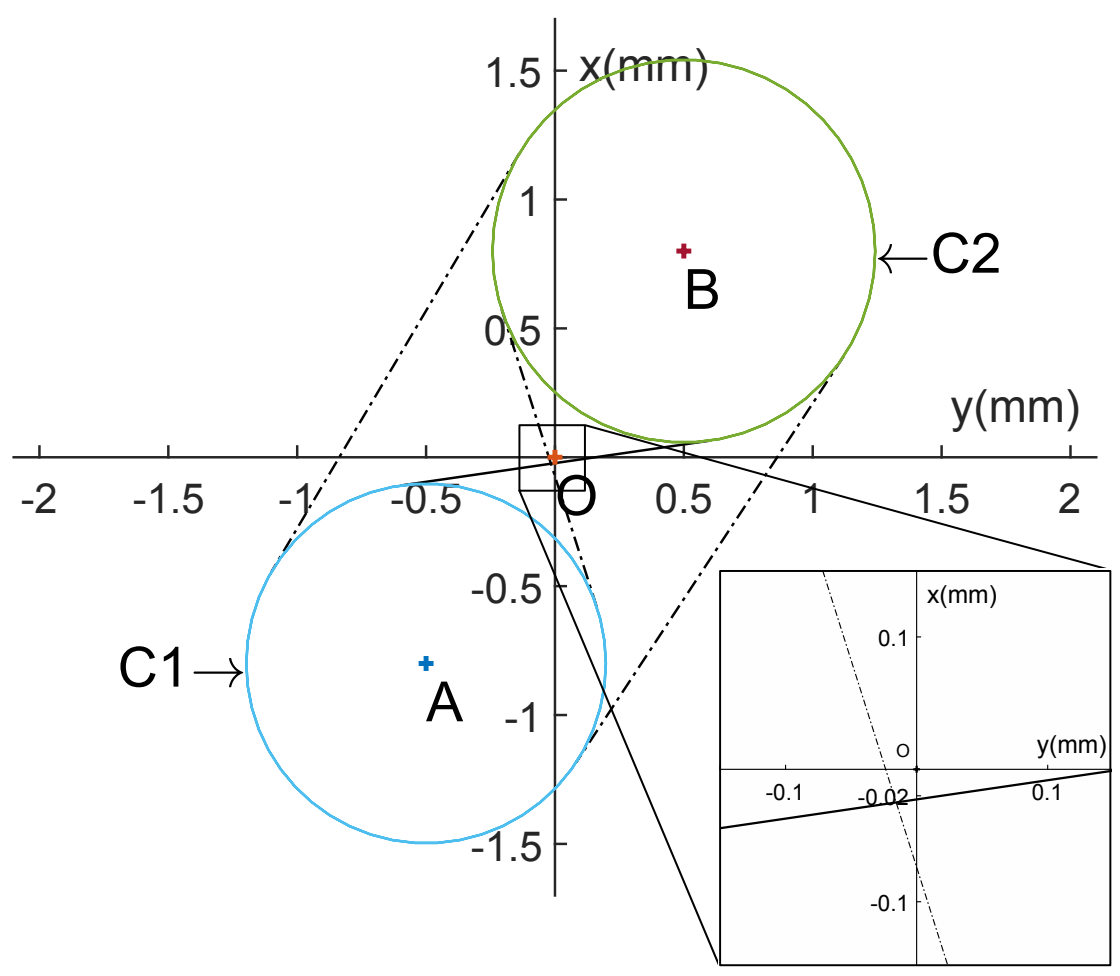

Figure 15: Four possible tube position and orientation reconstructions (three dash-dot lines and one solid line) located on the plane perpendicular to the insertion axis (parallel to the $x y$-plane) using two detection circle methods. The solid line is the most parallel line to the ground truth that is used to quantify the vessel reconstruction. Inset shows the intersection of the line at $x=-0.02$. O: tip of the PBN, A: position of the bottom probe, $\mathrm{B}$ : position of the top probe, $\mathrm{C} 1$ : the detection circle of the bottom probe, $\mathrm{C} 2$ : the detection circle of the top probe

\section{Discussion}

In this study, forward looking sensors have been implemented in a biologically inspired steerable needle, which borrows from the unique ovipositor design of certain wasps. The needle has the ability to steer around obstacles so that retraction and reinsertion is not needed, if a vessel is detected along its path. The needle is manufactured from a biocompatible, compliant material so it is suitable for long term brain treatment [3, 37]. As the focus of previous works in steerable needle sensing are position, force, and shape sensing $[18,24]$, the goal of this study was to develop embedded forward looking sensors that could detect a vessel in front of the tip of the needle and estimate its pose to inform an eventual obstacle avoidance strategy. 


\subsection{Characterization Results}

The characterization results illustrate the detection range of the $800 \mathrm{~nm}$ wavelength LDF probe. Using $5 \mathrm{~mm} / \mathrm{s}$ flow velocity in a tube with an inner diameter of $0.6 \mathrm{~mm}$ fulfills the minimum requirement to detect the vessel in front of the tip of a $2.5 \mathrm{~mm}$ OD PBN prototype, as it was shown to cater for up to $1 \mathrm{~mm}$ off-axis detection at 0.9 $\mathrm{mm}$ depth (Figure 4). One of the advantages of LDF is that it has a fast response time [52]. During insertion, an increase in the perfusion value means that the tip is approaching a vessel. If a high perfusion value is detected, the insertion can be stopped and, based on the vessel pose estimate, an avoidance strategy that complies with the needle's geometrical and steering constraints can be implemented. Using the sensors, needle insertion can be performed in a safer manner, allowing even vessels that may not have been visible in preoperative images, to be avoided. Indeed, the sensors can detect vessels with a diameter as small as $0.6 \mathrm{~mm}$, which may be hard to image using MRI, due to the limited resolution of the reconstructed volume (typically $0.59 \times 0.59 \times 1.50 \mathrm{~mm}$, as in [53]). Additionally, compared to MRI guided procedures, in which the patient has to be moved in and out of the scanner [54], our method and approach should reduce surgical time without compromising patient safety.

The measurement value of the laser Doppler system is affected by both the concentration of the scattering particles and the flow velocity. Figure 7 shows that a vessel with higher flow velocity can be detected further ahead compared to a lower velocity. Therefore, a vessel with lower flow velocity may not be detected before it is too close to the tip of the needle to initiate an avoidance procedure. Nonetheless, the $5 \mathrm{~mm} / \mathrm{s}$ flow velocity used here is half the value of the average blood flow velocity in a small vein $(10 \mathrm{~mm} / \mathrm{s})$ and a quarter of the blood flow velocity in an arteriole $(20 \mathrm{~mm} / \mathrm{s})$ [55]. It is therefore reasonable to believe that the system will work at least as well for more realistic physiological flow velocities. However, the size of the vessel also needs to be considered. In smaller vessels, the lower blood concentration inside it would reduce the perfusion value. Therefore, before surgical implementation, a minimum value of perfusion for vessels that must be avoided during surgery should be defined.

\subsection{Vessel Position and Orientation Prediction}

Here, two laser Doppler probes have been used to determine a safe region for the steerable needle to access in order to avoid a vessel sensed along its insertion path. Here, we have chosen to focus on a $5 \mathrm{~mm} / \mathrm{s}$ flow velocity inside a $0.6 \mathrm{~mm}$ inner diameter tube to prove the concept of LDF-based vessel detection. Due to a dependency on depth and flow velocity, it is not possible to determine the exact position of blood vessels with respect to the needle using a single measurement. Using two diagonally positioned laser Doppler probes in opposite PBN working channels shows the discrepancy between the perfusion values as a function of the distances between the vessel phantom and the probes. In Figure 13c, where the tip off-axis was set at $0 \mathrm{~mm}$, there is no significant difference between the perfusion values from the top and bottom probes, since the distance from 
the vessel phantom to each probe is equal. For other tip off-axis positions, significant differences are observed, starting at the maximum detection range of $2.1 \mathrm{~mm}$ away from the sensor. In a two dimensional world, where a tube/vessel has zero gradient with respect to the normal plane to the needle, the problem of vessel avoidance becomes one of moving on the plane, and thus the vessel can be identified and avoided. However, the vessel angle is in the range of $0^{\circ} \pm 180^{\circ}$ to the needle's normal plane, and thus further data processing is required.

Successive measurements using at least three data points have been used to determine the distance from the tip of the needle to the tube surface and the possible tip off-axis position. Successive measurements rely on the accuracy of the inverse of (1). Equation 1 was derived empirically, since understanding the random scattering inside a medium like brain tissue is commonly achieved using simulation results based on random walks $[44,56]$. In this paper, (1) was only used to smoothen the characterization data.

Using two probes, the distance from the tip to the needle is determined by the mean of the distance from the top and the bottom probes. The average distance prediction from the probe to the surface of the tube gives sub-millimeter accuracy, with an RMS error of $0.37 \mathrm{~mm}$. However the RMS error of the angle measurement is $15.6^{\circ}$. The effect of the tip geometry on tissue deformation has not been considered in the prediction algorithm presented here, and this may be the main source of this rotational inaccuracy. However, for the steerable needle system, in order to avoid an obstacle (e.g. a vessel), the avoidance direction can be set at $90^{\circ}$ to the direction of the vessel axis. If this avoidance direction is used, it is still safe to avoid the vessel, even with the angular RMS error measured here. The algorithm outputs four possible poses for the vessel, and thus the needle "escape" direction should be set as the one which would result in the smallest risk to puncture any of these. As this method is believed to be the first method to employ embedded optical fibers to predict vessel pose, no comparison in terms of accuracy of the system can be made with the existing literature. In future work, four LDF probes will be used, since, in theory, this should eliminate any possible ambiguity, cutting down the number of possible vessel configurations to one.

It should be noted that the detection range of the sensor is only $2.1 \mathrm{~mm}$ from the needle tip. The detection range can be increased by using a sensor with a longer wavelength (currently the system uses an $800 \mathrm{~nm}$ laser). However, using an optical system, the longest detection range that is found in the literature is $3-4 \mathrm{~mm}$ [13]. This is less than the minimum radius of curvature of our latest PBN design (a detection depth of $15 \mathrm{~mm}$ is needed for a needle with $70 \mathrm{~mm}$ radius of curvature, assuming the vessel to be avoided has a diameter of $1 \mathrm{~mm}$, with $0 \mathrm{~mm}$ tip-off-axis position). Therefore, a short retraction, followed by reinsertion may be required to completely avoid the vessel, depending on its predicted orientation. Even though retraction is required, full extraction and reinsertion can be avoided, which would be the only option for rigid needle designs, and thus what is proposed here offers a clear benefit for the patient.

The inverse data computed from the perfusion value of depth and off-axis positions used in this paper is only valid for the given combination of vessel, medium properties 
and sensing configuration. Any change in the flow velocity rates, tube diameter, optical properties of the tissue phantom, and the type of the sensor, may change the relationship of the empirical inverse function described here. Since it has been shown that vessel direction can be predicted using multiple probes combined with successive measurements, finding a more complete relationship for every parameter is possible and will be part of future work. A multi-layer inverse function including, for example, variable flow velocity rates, tube sizes, and tissue optical properties, could be used to give a probability for vessel prediction with a certain confidence interval. Even though laser Doppler probes were used in this paper, the concept of finding a possible vessel orientation using two detection circles can be ported to other imaging modalities, such as OCT or CGD using a multi-core fiber.

Finally, in this proof of concept, static experiments were performed. In future work, real-time measurements while the probe is moving will be conducted. This requires advanced signal processing to discriminate between tissue motions and a blood vessel, since both of these must be simultaneously detected.

In summary, the method for vessel pose prediction presented here makes several novel contributions: (1) the use of relative measurements between multiple laser Doppler probes to predict which probe is closer to the vessel; (2) the use of successive laser Doppler measurements to determine the likely depth and off-axis position of the vessel; (3) the use of two laser Doppler probes to infer the likely vessel pose.

\section{Conclusion}

This paper proposes a novel vessel detection method for steerable needle systems with multiple lumens. The specific proof-of-concept implementation described here employs two laser Doppler flowmetry probes, embedded withing a unique multi-segment, multilumen design of the PBN, a steerable device inspired by the egg laying channel of certain parasitic wasps. Errors of $0.37 \mathrm{~mm}$ (RMS) in depth detection and $15.6^{\circ}$ angular error for predicting the vessel pose were achieved here, but with significant potential for improvement. Indeed, better knowledge of the effect of tip geometry on sensor measurements and the deployment of additional sensors within the PBN (e.g. 4 laser Doppler probes, which could be deployed without any change to the needle design) would improve the performance of the system considerably. Associating such a comprehensive detection system with appropriate obstacle avoidance control strategies is expected to reduce the risk of hemorrhage in keyhole neurosurgery, thereby paving the way for the eventual clinical application of safer surgical needle steering technology.

\section{Acknowledgments}

Acknowledgment This research was supported by the Indonesian Directorate General of Higher Education and the European Research Council under the European Unions Seventh Framework Programme (FP7/20072013) / ERC grant agreement no 258642- 
STING, and EPSRC. This project has also received funding from the European Unions Horizon 2020 research and innovation programme under grant agreement 688279. The authors are grateful to Michael Rau and Oxford Optronics for the loan of the equipment and advice relating to its use.

\section{References}

[1] D Pinggera, I Kvitsaridtze, G Stockhammer, W Eisner, C Thomé, CF Freyschlag, P Moser, and $\mathrm{J}$ Kerschbaumer. Serious tumor seeding after brainstem biopsy and its treatmenta case report and review of the literature. Acta neurochirurgica, 159(4):751-754, 2017.

[2] Aparna Wagle Shukla and Michael S Okun. State of the art for deep brain stimulation therapy in movement disorders: A clinical and technological perspective. IEEE reviews in biomedical engineering, 9:219-233, 2016.

[3] Raghu Raghavan, Martin L Brady, María Inmaculada Rodríguez-Ponce, Andreas Hartlep, Christoph Pedain, and John H Sampson. Convection-enhanced delivery of therapeutics for brain disease, and its optimization. Neurosurgical focus, 20(4):E12, 2006.

[4] Devin K Binder, Geoff Rau, and Philip A Starr. Hemorrhagic complications of microelectrodeguided deep brain stimulation. Stereotactic and functional neurosurgery, 80(1-4):28-31, 2003.

[5] M Field, T F Witham, J C Flickinger, D Kondziolka, and L D Lunsford. Comprehensive assessment of hemorrhage risks and outcomes after stereotactic brain biopsy. Journal of Neurosurgery, 94(4):545-551, 2001.

[6] Albert J Fenoy and Richard K Simpson. Risks of common complications in deep brain stimulation surgery: management and avoidance. Journal of neurosurgery, 120(January):132-9, 2014.

[7] Graeme F Woodworth, Matthew J McGirt, Amer Samdani, Ira Garonzik, Alessandro Olivi, and Jon D Weingart. Frameless image-guided stereotactic brain biopsy procedure: diagnostic yield, surgical morbidity, and comparison with the frame-based technique. Journal of neurosurgery, 104(2):233-237, 2006.

[8] Walter A Hall. The safety and efficacy of stereotactic biopsy for intracranial lesions. Cancer, 82(9):1749-1755, 1998.

[9] Atsushi Umemura, Jurg L Jaggi, Howard I Hurtig, Andrew D Siderowf, Amy Colcher, Matthew B Stern, and Gordon H Baltuch. Deep brain stimulation for movement disorders: morbidity and mortality in 109 patients. Journal of neurosurgery, 98(4):779-784, 2003.

[10] Ian J Gerard, Marta Kersten-Oertel, Kevin Petrecca, Denis Sirhan, Jeffery A Hall, and D Louis Collins. Brain shift in neuronavigation of brain tumors: A review. Medical image analysis, 35:403-420, 2017.

[11] Terry Peters and Kevin Cleary. Image-guided interventions: technology and applications. Springer Science \& Business Media, 2008.

[12] Michael I Miga. Computational modeling for enhancing soft tissue image guided surgery: an application in neurosurgery. Annals of biomedical engineering, 44(1):128-138, 2016.

[13] Chia-Pin Liang, Yalun Wu, Joe Schmitt, Paul E Bigeleisen, Justin Slavin, M Samir Jafri, Cha-Min Tang, and Yu Chen. Coherence-gated Doppler: a fiber sensor for precise localization of blood flow. Biomed. Opt. Express, 4(5):760-71, 2013.

[14] Chia-Pin Liang, Jeremiah Wierwille, Thais Moreira, Gary Schwartzbauer, M. Samir Jafri, Cha-Min Tang, and Yu Chen. A forward-imaging needle-type OCT probe for image guided stereotactic procedures. Optics Express, 19(27):26283-26294, 2011.

[15] Karin Wårdell, Simone Hemm-Ode, Peter Rejmstad, and Peter Zsigmond. High-Resolution Laser Doppler Measurements of Microcirculation in the Deep Brain Structures: A Method for Potential Vessel Tracking. Stereotactic and Functional Neurosurgery, pages 1-9, 2016.

[16] Niki Abolhassani, Rajni Patel, and Mehrdad Moallem. Needle insertion into soft tissue: A survey. Medical engineering 83 physics, 29(4):413-431, 2007. 
[17] Nick J van de Berg, Dennis J van Gerwen, Jenny Dankelman, and John J van den Dobbelsteen. Design choices in needle steeringa review. IEEE/ASME Transactions on Mechatronics, 20(5):2172-2183, 2015.

[18] Ann Majewicz, Joshua J Siegel, Andrew A Stanley, and Allison M Okamura. Design and evaluation of duty-cycling steering algorithms for robotically-driven steerable needles. In 2014 IEEE International Conference on Robotics and Automation (ICRA), pages 5883-5888. IEEE, 2014.

[19] Hunter B Gilbert, Joseph Neimat, and Robert J Webster. Concentric tube robots as steerable needles: Achieving follow-the-leader deployment. IEEE Transactions on Robotics, 31(2):246$258,2015$.

[20] Nick J van de Berg, Jenny Dankelman, and John J van den Dobbelsteen. Design of an actively controlled steerable needle with tendon actuation and fbg-based shape sensing. Medical engineering $\mathcal{E}$ physics, 37(6):617-622, 2015.

[21] Troy K Adebar, Joseph D Greer, Paul F Laeseke, Gloria L Hwang, and Allison M Okamura. Methods for improving the curvature of steerable needles in biological tissue. IEEE Transactions on Biomedical Engineering, 63(6):1167-1177, 2016.

[22] Seong Young Ko, Luca Frasson, and Ferdinando Rodriguez y Baena. Closed-loop planar motion control of a steerable probe with a programmable bevel inspired by nature. IEEE Transactions on Robotics, 27(5):970-983, 2011.

[23] Arnau Garriga Casanovas and F Rodriguez y Baena. Complete follow-the-leader kinematics using concentric tube robots. the International Journal of Robotics Research, to appear.

[24] Carlos Rossa and Mahdi Tavakoli. Issues in closed-loop needle steering. Control Engineering Practice, 62:55-69, 2017.

[25] Troy K Adebar, Ashley E Fletcher, and Allison M Okamura. 3-d ultrasound-guided robotic needle steering in biological tissue. IEEE Transactions on Biomedical Engineering, 61(12):2899-2910, 2014.

[26] Momen Abayazid, Marco Kemp, and Sarthak Misra. 3d flexible needle steering in soft-tissue phantoms using fiber bragg grating sensors. In 2013 IEEE International Conference on Robotics and Automation (ICRA), pages 5843-5849. IEEE, 2013.

[27] Elif Ayvali, Chia-Pin Liang, Mingyen Ho, Yu Chen, and Jaydev P Desai. Towards a discretely actuated steerable cannula for diagnostic and therapeutic procedures. The International journal of robotics research, 31(5):588-603, 2012.

[28] L Frasson, T Parittotokkaporn, A Schneider, BL Davies, JFV Vincent, SE Huq, P Degenaar, and FM Rodriguez Baena. Biologically inspired microtexturing: Investigation into the surface topography of next-generation neurosurgical probes. In 2008 30th Annual International Conference of the IEEE Engineering in Medicine and Biology Society, pages 5611-5614. IEEE, 2008.

[29] Donald L. J. Quicke. The Braconid and Ichneumonid Parasitoid Wasps: Biology, Systematics, Evolution and Ecology. Wiley-Blackwell, web edition, 2014.

[30] Aimée Sakes, Dimitra Dodou, and Paul Breedveld. Buckling prevention strategies in nature as inspiration for improving percutaneous instruments: a review. Bioinspiration 83 biomimetics, 11(2):021001, 2016.

[31] JFV Vincent and MJ King. The mechanism of drilling by wood wasp ovipositors. Biomimetics (USA), 1995.

[32] Matthew J Oldfield, Alexander Leibinger, Tian En Timothy Seah, and Ferdinando Rodriguez y Baena. Method to Reduce Target Motion Through NeedleTissue Interactions. Annals of Biomedical Engineering, 2015.

[33] Uroš Cerkvenik, Bram van de Straat, Sander WS Gussekloo, and Johan L van Leeuwen. Mechanisms of ovipositor insertion and steering of a parasitic wasp. Proceedings of the National Academy of Sciences, 114(37):E7822-E7831, 2017.

[34] Ram Gal, Maayan Kaiser, Gal Haspel, and Frederic Libersat. Sensory arsenal on the stinger of the parasitoid jewel wasp and its possible role in identifying cockroach brains. PloS one, 
9(2):e89683, 2014.

[35] Christopher Burrows, Riccardo Secoli, and Ferdinando Rodriguez y Baena. Experimental characterisation of a biologically inspired 3d steering needle. In 2013 13th International Conference on Control, Automation and Systems (ICCAS), pages 1252-1257. IEEE, 2013.

[36] Hunter B Gilbert and Robert J Webster. Can concentric tube robots follow the leader? In 2013 IEEE International Conference on Robotics and Automation (ICRA), pages 4881-4887. IEEE, 2013.

[37] Kathryn H Rosenbluth, Matthias Luz, Erich Mohr, Stephan Mittermeyer, John Bringas, and Krystof S Bankiewicz. Design of an in-dwelling cannula for convection-enhanced delivery. Journal of neuroscience methods, 196(1):118-123, 2011.

[38] L Frasson, J Neubert, S Reina, M Oldfield, BL Davies, and F Rodriguez y Baena. Development and validation of a numerical model for cross-section optimization of a multi-part probe for soft tissue intervention. In Engineering in Medicine and Biology Society (EMBC), 2010 Annual International Conference of the IEEE, pages 3202-3205. IEEE, 2010.

[39] Luca Frasson, Francesco Ferroni, Seong Young Ko, Gorkem Dogangil, and Ferdinando Rodriguez y Baena. Experimental evaluation of a novel steerable probe with a programmable bevel tip inspired by nature. Journal of robotic surgery, 6(3):189-197, 2012.

[40] Alexander Leibinger, Matthew Oldfield, and Ferdinando Rodriguez y Baena. Multi-objective design optimization for a steerable needle for soft tissue surgery. In The 15th International Conference on Biomedical Engineering, pages 420-423. Springer, 2014.

[41] M Scali, D Kreeft, P Breedveld, and Dimitra Dodou. Design and evaluation of a wasp-inspired steerable needle. In Ral J. Martn-Palma Mato Knez, Akhlesh Lakhtakia, editor, Bioinspiration, Biomimetics, and Bioreplication 2017, pages 1016207-1016207. International Society for Optics and Photonics, 2017.

[42] Ingemar Fredriksson, Marcus Larsson, and Tomas Strömberg. Model-based quantitative laser doppler flowmetry in skin. Journal of biomedical optics, 15(5):057002-057002, 2010.

[43] A Liebert, M Leahy, and R Maniewski. Multichannel laser-doppler probe for blood perfusion measurements with depth discrimination. Medical and Biological Engineering and Computing, 36(6):740-747, 1998.

[44] Ingemar Fredriksson, Marcus Larsson, and Tomas Strömberg. Measurement depth and volume in laser Doppler flowmetry. Microvascular Research, 78(1):4-13, 2009.

[45] Chien Hsiang Huang, Chiao Chi V Chen, Tiing Yee Siow, Sheng Hsiou S Hsu, Yi Hua Hsu, Fu Shan Jaw, and Chen Chang. High-resolution structural and functional assessments of cerebral microvasculature using 3D gas $\Delta \mathrm{R} 2^{*}$-mMRA. PloS One, 8(11), 2013.

[46] AN Yaroslavsky, PC Schulze, IV Yaroslavsky, R Schober, F Ulrich, and HJ Schwarzmaier. Optical properties of selected native and coagulated human brain tissues in vitro in the visible and near infrared spectral range. Physics in medicine and biology, 47(JULY):2059-2073, 2002.

[47] Brian W Pogue, Lothar Lilge, Michael S Patterson, Brian C Wilson, and Tayyaba Hasan. Absorbed photodynamic dose from pulsed versus continuous wave light examined with tissue-simulating dosimeters. Applied Optics, 36(28):7257-7269, 1997.

[48] Christopher Burrows, Fangde Liu, and Ferdinando Rodriguez y Baena. Smooth on-line path planning for needle steering with non-linear constraints. In 2015 IEEE/RSJ International Conference on Intelligent Robots and Systems (IROS), pages 2653-2658. IEEE, 2015.

[49] Giulia Franceschini. The mechanics of human brain tissue. Modelling, preservation and control of materials and structures, University of Trento, 2006.

[50] P Å Öberg. Tissue motion - a disturbance in the laser-Doppler blood flow signal? Technology and Health Care, 7(2-3):185-92, 1999.

[51] Lihong Wang, Steven L Jacques, and Liqiong Zheng. Mcmlmonte carlo modeling of light transport in multi-layered tissues. Computer methods and programs in biomedicine, 47(2):131-146, 1995.

[52] Vinayakrishnan Rajan, Babu Varghese, Ton G van Leeuwen, and Wiendelt Steenbergen. Review of methodological developments in laser doppler flowmetry. Lasers in medical science, 24(2):269- 
283, 2009 .

[53] Silvain Bériault, Abbas F Sadikot, Fahd Alsubaie, Simon Drouin, D Louis Collins, and G Bruce Pike. Neuronavigation using susceptibility-weighted venography: application to deep brain stimulation and comparison with gadolinium contrast. Journal of neurosurgery, 121(1):131$141,2014$.

[54] Karl A Sillay, Deborah Rusy, Laura Buyan-Dent, Nancy L Ninman, and Karl K Vigen. Wide-bore $1.5 \mathrm{t}$ mri-guided deep brain stimulation surgery: initial experience and technique comparison. Clinical neurology and neurosurgery, 127:79-85, 2014.

[55] Ingemar Fredriksson. Quantitative Laser Doppler Flowmetry. Number 1269. 2009.

[56] Ricky Hennessy, Will Goth, Manu Sharma, Mia K Markey, and James W Tunnell. Effect of probe geometry and optical properties on the sampling depth for diffuse reflectance spectroscopy. Journal of biomedical optics, 19(10):107002-107002, 2014. 